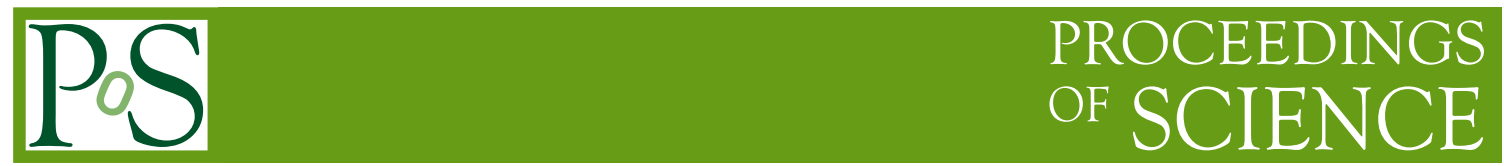

\title{
Experimental Results on Soft Strong Interactions
}

\section{Paul Newman*}

School of Physics \& Astronomy, The University of Birmingham, B15 2TT, UK.

E-mail: p.r.newman@bham.ac.uk

\begin{abstract}
A brief report is given on selected recent highlights from experimental studies of soft and semihard strong interactions from the Large Hadron Collider, HERA and the Tevatron. The subject is broken down into diffractive and non-diffractive channels and summarises the most significant recent progress and open questions in each area.
\end{abstract}

36th International Conference on High Energy Physics,

July 4-11, 2012

Melbourne, Australia

*Speaker. 


\section{Introduction}

The Melbourne ICHEP 2012 conference will remain in the memories of all who attended, as the meeting where the Higgs boson discovery was announced and the first information on its properties began to emerge. Although it is also concerned with the study of the new energyfrontier physics landscape offered by the Large Hadron Collider (LHC), the material covered in this contribution could hardly be more different: whereas a Standard Model Higgs boson with a mass of around $125 \mathrm{GeV}$ is produced once in around $10^{10} \mathrm{pp}$ collisions, soft strong interactions are at work in almost all LHC collisions. Paradoxically, our understanding of the processes at work in these most ordinary of processes is much poorer than is the case for Higgs production. Whilst availability of data is not an issue, the complexity of the mechanisms involved and the lack of a precision theory in the absence of a large momentum or energy scale make soft strong interactions far less predictable and more poorly understood than the physics of the Higgs sector. The study of the bulk of the LHC cross section under what are often referred to as 'minimum bias' conditions is therefore in a very real sense, virgin 'swagman in billabong' [1] territory. In addition to the new measurements for the LHC, our models of soft strong interactions ${ }^{1}$ have also recently been constrained by data from HERA and the Tevatron. In addition to improved precision on previously measured quantities, new observables with enhanced or complementary sensitivity to the underlying dynamics have also been introduced.

There are fundamental reasons why it is important to understand soft strong interactions better. It is the non-perturbative strong force which binds the fundamental quarks and gluons inside hadrons, the detailed mechanisms for which remain poorly understood. In addition to this question of confinement, soft strong interactions are at the heart of the dynamical generation of hadronic mass. There may also be deep connections through string theory [5] between the soft hadronic degrees of freedom which drive the strong interaction at large coupling strengths and the weakly coupled regime of gravity. At a more practical level, it is the minimum bias processes which are most pertinent to the modelling of background activity in the LHC detectors caused by the pileup of multiple events in the same bunch crossing. Even within a single collision, the background activity caused by multiple interactions and the underlying event requires an understanding of minimum bias physics. There are also many applications beyond the LHC, for example in modelling the development of cosmic ray air showers [6].

This talk [7] naturally covered not only soft interactions, but also the interface between the soft and hard regimes, which is a natural starting point for attempts to make a complete description of strong interactions. Hadron-hadron collisions are broken down into broad categories as illustrated for the case of $p p$ scattering in figure 1. The simplest process is elastic scattering ( $p p \rightarrow p p$, figure 1a), in which the two incoming protons collide at a centre of mass energy $\sqrt{ } s$ and remain intact. This process can be described by a single non-trivial variable, which is usually chosen to be the squared four-momentum transfer, $t$. Beyond the elastic case, the next simplest process is single diffractive dissociation ( $p p \rightarrow X p$, figure $1 \mathrm{~b}$ ), in which one of the beam particles dissociates to produce a multi-particle excitation $X$, possessing a continuum of masses $M_{X}$. In addition to $t$, such processes are described by a further invariant, which may be chosen to be either $M_{X}$, or the fractional energy loss of the intact proton $\xi=M_{X}^{2} / s$. In the closely related double diffractive

\footnotetext{
${ }^{1}$ The most commonly used examples up to now are PYTHIA6 [2], PYTHIA8 [3] and PHOJET [4].
} 


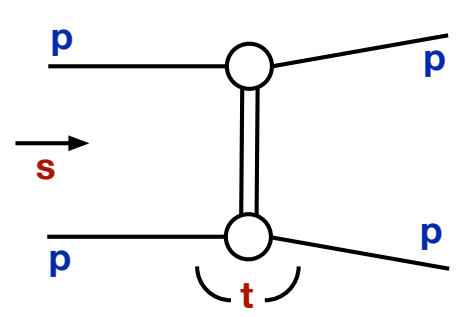

(a)

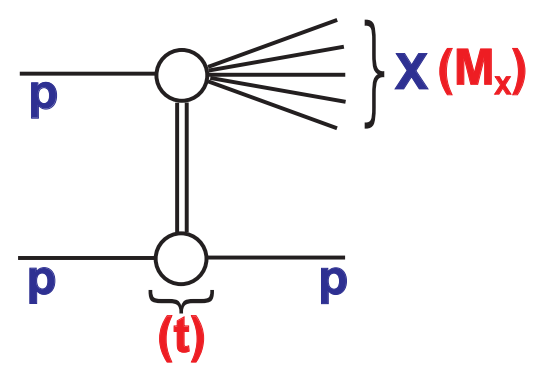

(b)

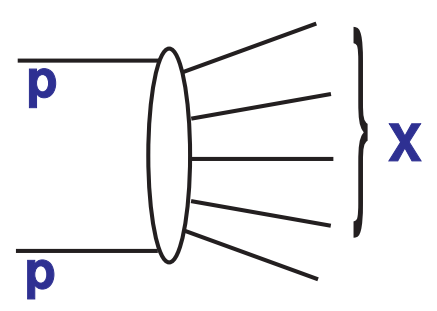

(c)

Figure 1: Schematic illustrations of (a) the elastic process $p p \rightarrow p p$, (b) the single diffractive dissociation process $p p \rightarrow X p$ and (c) the non-diffractive process $p p \rightarrow X$. The kinematic variables discussed in the text are indicated.

dissociation process ( $p p \rightarrow X Y$ ), both beam particles dissociate to produce two independently fragmenting and hadronising systems $X$ and $Y$. For LHC kinematics, the dissociation masses $M_{X}$ and $M_{Y}$ may be as large as $1 \mathrm{TeV}$. The final, non-diffractive category ( $p p \rightarrow X$, figure 1c), includes all processes not described by the elastic and diffractive channels. In the non-diffractive case, particle production typically takes place throughout the full available rapidity region. Crudely, about half of the total $p p$ cross section at LHC energies is attributable to non-diffractive processes, with the remainder attributable to the diffractive contributions.

\section{Elastic Scattering and Total Cross Sections}

The elastic scattering cross section has recently been measured differentially in $t$ both by D0 at the Tevatron [8] and by TOTEM at the LHC [9] (see figure 2a). As has been the case historically, the low $t$ region is well modelled by an exponential dependence, $\mathrm{d} \sigma / \mathrm{d} t \propto \exp (B t)$, such that the slope parameter, $B$ quantifies the spatial extent of the interaction region. The recent results $(B=$ $16.9 \pm 0.2 \mathrm{GeV}^{-2}$ at $\sqrt{ } s=1.96 \mathrm{TeV}$ [8] and $B=19.9 \pm 0.3 \mathrm{GeV}^{-2}$ at $\sqrt{ } s=7 \mathrm{TeV}$ [9]) confirm that the slope parameter increases with energy (shrinkage of the forward elastic peak) and suggest a faster growth than was obtained at lower energy and is usually assumed in models. A possible interpretation of this lies in process and energy-dependent absorptive corrections. The increase in $B$ and the decrease of the point in $|t|$ at which the characteristic minimum in the distribution occurs (from around $|t|=0.7 \mathrm{GeV}^{2}$ at the Tevatron to around $0.53 \mathrm{GeV}^{2}$ at the $\mathrm{LHC}$ ) both indicate that the effective size of the region over which the protons interact grows with energy, as might be expected as longer and longer-lived quantum fluctuations become important.

It is interesting to compare these results with those from quasi-elastic vector meson production at HERA, where, in the case where a hard scale is provided by either $Q^{2}$ or the quark composition of the vector meson, the size of the interaction region corresponds to that of the proton alone. Recent results, including a first measurement of $\Upsilon$ production, have yielded $B \sim 5 \mathrm{GeV}^{-2}$, suggesting a rather small effective proton size of around $0.6 \mathrm{fm}$ [13]. Given that vector meson photoproduction is driven at lowest order by two gluon exchange, this in turn indicates that the gluon radius of the proton may be smaller than its quark radius, which is well measured from electromagneitc processes to be around $0.8 \mathrm{fm}$. 


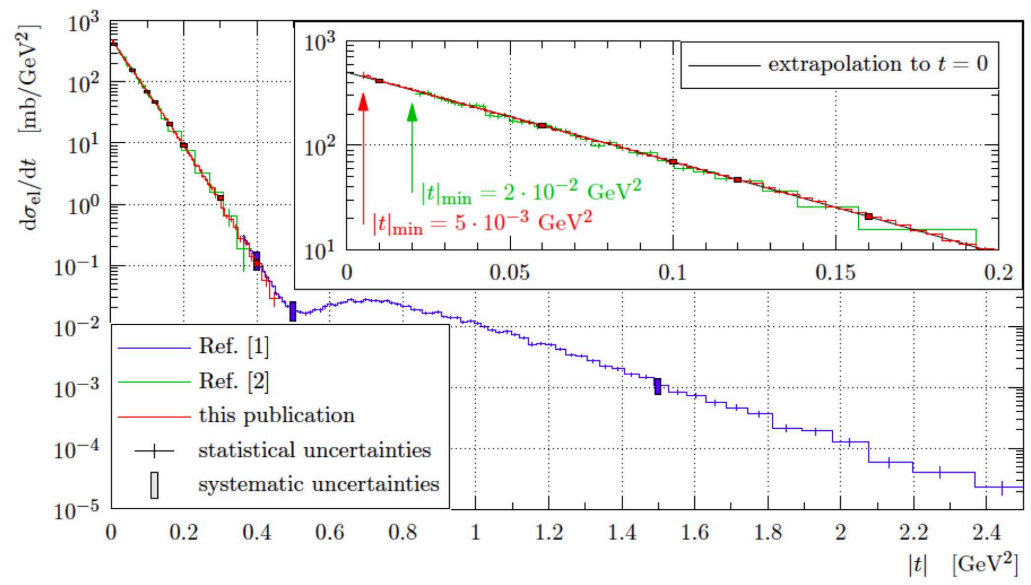

(a)

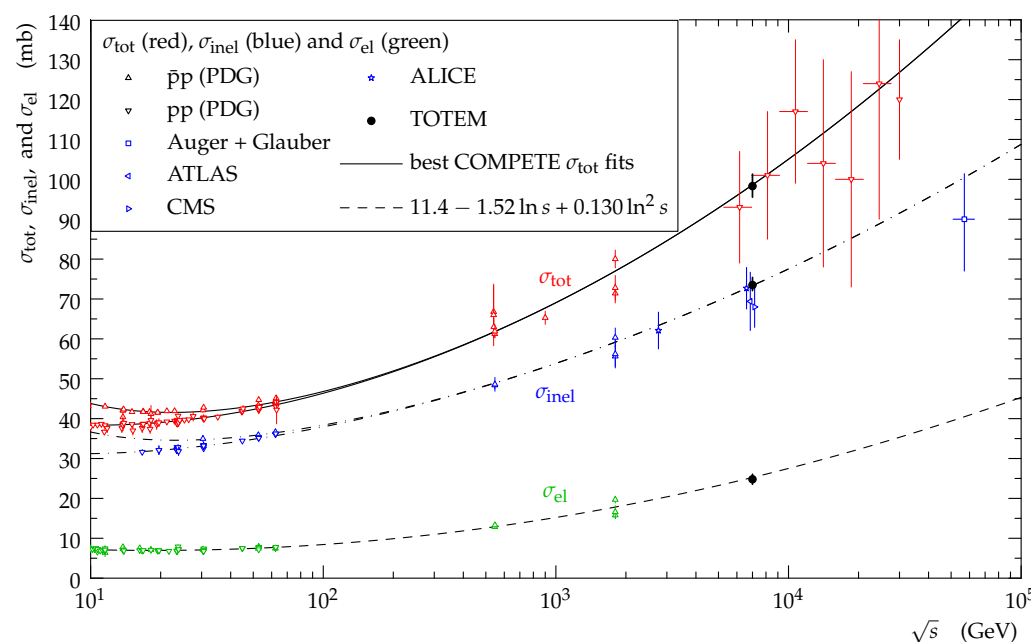

(b)

Figure 2: (a) Compilation of TOTEM elastic scattering results [9]. The data indicated as 'Ref 1' and 'Ref 2' and the curve denoted 'this publication' correspond, respectively, to citations [10], [11] and [9] here. (b) Compilation of total, total-inelastic and elastic cross section data [11]. Additional results at $\sqrt{s}=8 \mathrm{TeV}$ have also recently appeared [12].

Elastic cross sections at $t \rightarrow 0$ are closely related to total cross sections, via the optical theorem. The TOTEM collaboration have exploited this fact to extract the total $p p$ cross section at LHC energies in several ways, including one method which is independent of luminosity measurements [12]. In the example shown in figure $2 b$, the total cross section is obtained via

$$
\sigma_{\mathrm{tot}}^{2}=\frac{16 \pi}{1+\rho^{2}}\left(\frac{\mathrm{d} \sigma_{\mathrm{el}}}{\mathrm{d} t}\right)_{t=0} .
$$

The result is consistent with either a logarithmic or a power law rise with $s$ relative to previous data. Subtracting the elastic from the total cross section yields the total inelastic cross section, which is also shown in figure 2b. This quantity has been measured directly by ATLAS [14], ALICE [15] 
and CMS [16] in addition to the indirect TOTEM extraction, with consistent results obtained.

\section{Soft Diffractive Dissociation}

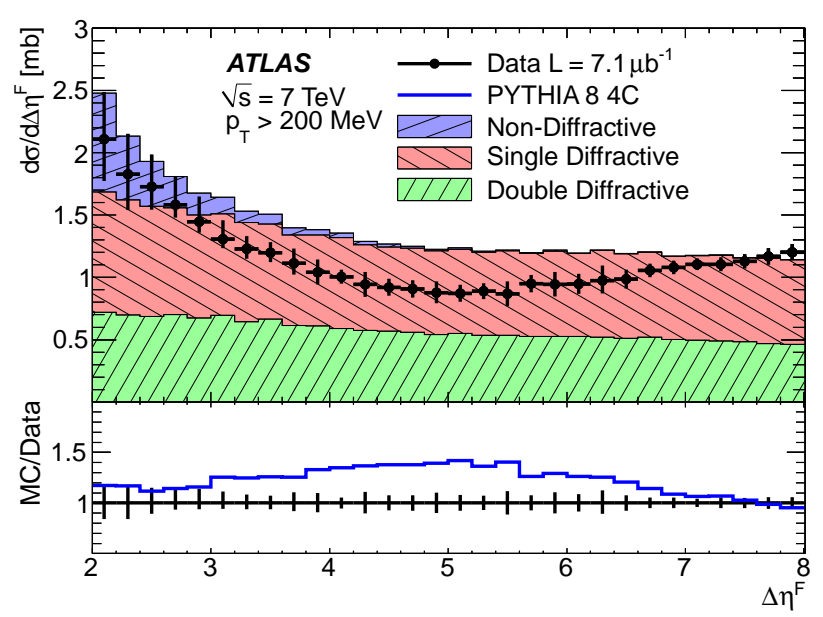

Figure 3: Cross section for LHC events containing large rapidity gaps, extending from at least $\eta= \pm 4.9$ to $\eta= \pm 4.9 \mp \Delta \eta_{F}$ [17]. For the contribution from diffractive dissociation processes, the left hand side of the plot corresponds approximately to $\xi=10^{-2.5}$ and the right hand side to $\xi=10^{-5}$. The data are compared with the predictions of an the PYTHIA8 Monte Carlo model [3], tuned using inclusive ATLAS minimum bias data.

Predictions for inelastic diffraction at LHC energies varied substantially before the first data were taken. The first detailed measurement of the dynamics of the process, by ATLAS [17], exploits the close correlation between $\xi$ and the size $\Delta \eta$ of the rapidity gap separating the system $X$ form the intact leading proton $(\Delta \eta \approx-\ln \xi)$. Since the coverage of the central components of the detector is restricted to $|\eta|<4$.9, the chosen experimental observable, $\Delta \eta^{F}$, measures the size of the gap relative to $\eta= \pm 4$.9. At large gap sizes $\Delta \eta^{F} \lesssim 2$, the differential cross section $\mathrm{d} \sigma / \mathrm{d} \Delta \eta^{F}$ is approximately constant as a function of gap size (figure 3), as expected where diffractive processes dominate. When viewed in detail, the size of the cross section and its residual dependence on $\Delta \eta^{F}$ are sensitive to the dynamics of soft diffraction, the relative importance of the single and double diffractive dissociation cross sections and the role of absorptive corrections [18].

\section{Partonic Structure of Diffractive Dissociation}

The study of diffractive processes has been a major theme at the HERA electron-proton collider, where in addition to soft processes, hard and semi-hard interactions can be investigated, involving hard scales provided by the photon virtuality $Q^{2}$ or large transverse momenta generated in jets (figures $4 \mathrm{a}$ and $4 \mathrm{~b}$, respectively). With the most recent diffractive DIS (DDIS) data, close agreement has developed between the H1 and ZEUS measurements. A first combination of inclusive diffractive data from the two experiments, using measurements obtained by tagging and measuring the leading protons emerging intact from the interactions, has recently been published 


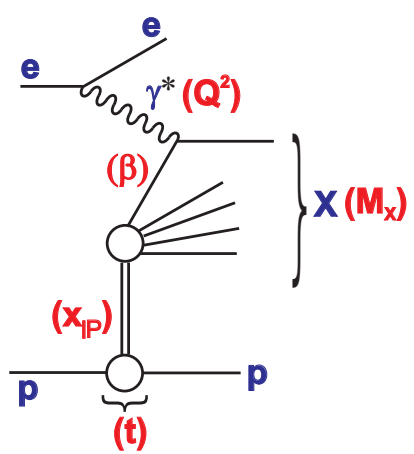

(a)

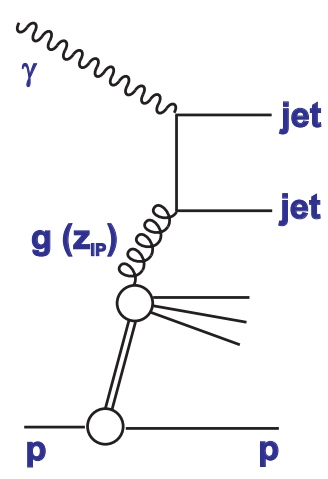

(b)

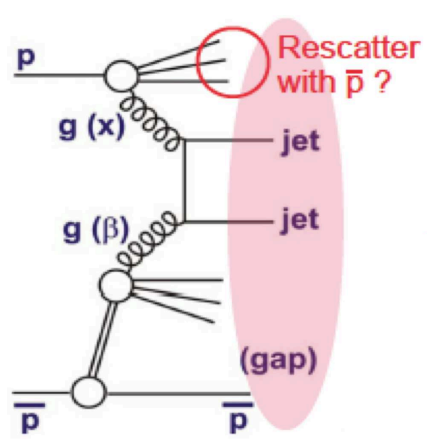

(c)

Figure 4: Sketches of diffractive hard scattering processes. (a) Inclusive DDIS at the level of the quark parton model, illustrating the commonly used kinematic variables. (b) Dominant lowest order diagram for high transverse momentum jet production in DDIS, in which a parton of momentum fraction $z_{\mathbb{P}}$ from the DPDFs enters the hard scattering. (c) Diffractive dijet production in $\bar{p} p$ scattering, illustrating the rescattering mechanism which is believed to produce a rapidity gap survival probability significantly smaller than unity.

[19]. The combination is shown for a selection of illustrative data points in figure 5. The improvement in precision is considerably better than would be expected on statistical grounds alone, due to the effective cross-calibration between the two measurements implicit in the averaging procedure.

The inclusive diffractive cross sections, often together with diffractive dijet data, are subjected to standard next-to-leading-order QCD fits based on DGLAP evolution, to obtain diffractive parton densities (DPDFs), corresponding to conditional probability distributions for partons of different flavours to be present in the proton at particular momentum fractions (denoted $z$ or $z_{\mathbb{P}}$ ) under the constraint that the proton stays intact with a given $\xi$. Recent examples are shown in figure 6 [20]. These results confirm, with improved precision, previous conclusions [21] that the DPDFs are dominated by a gluon density, which extends to large momentum fractions.

DPDFs such as those shown in figure 6 have been applied as an input to QCD calculations of a wide range of diffractive processes within DIS. Such comparisons have proved to be universally successful. A recent example [22] is shown in figure 7. Here, for the first time at HERA, dijet cross sections are measured in diffractive events selected on the basis of an intact reconstructed final state proton. Using this selection method in place of rapidity gap based techniques allows the study of events in which one of the reconstructed jets is close in rapidity to the edge of the rapidity gap. This topology is sensitive to possible hard diffractive production, where the full exchanged momentum enters into the hard subprocess producing the jets, a process which ought not to be described by the DPDF approach. The good agreement in figure 7 indicates that the hard diffractive process represents only a relatively small contribution throughout the accessible kinematic range.

Whilst models based on DPDFs work well to describe all diffractive dissociation processes in DIS, they fail spectacularly (by a factor of around 10) when DPDFs extracted from HERA data are applied to diffractive $\bar{p} p$ scattering at the Tevatron [23]. This discrepancy is usually interpreted in terms of multiple scattering effects, which occur in the presence of beam remnants (figure 4c). 


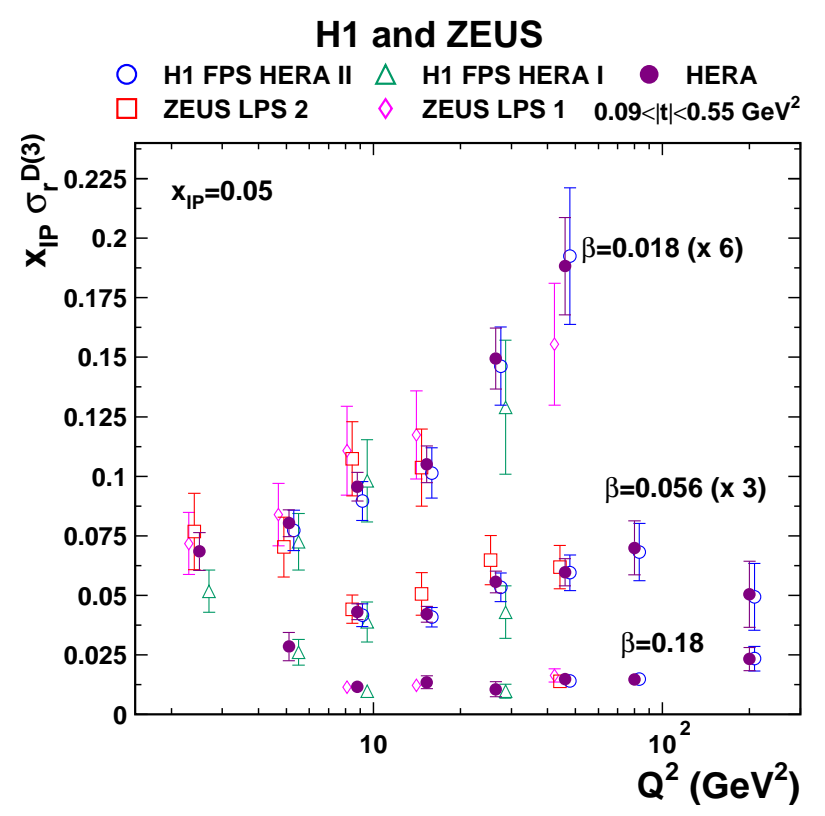

Figure 5: $Q^{2}$ dependence of the diffractive DIS cross section at $x_{I P} \equiv \xi=0.05$ and selected values of $\beta=x / x_{I P}$. Results are shown from H1 and ZEUS measurements in which leading protons are tagged in dedicated spectrometers well downstream of the interaction point, as well as their combination [19]
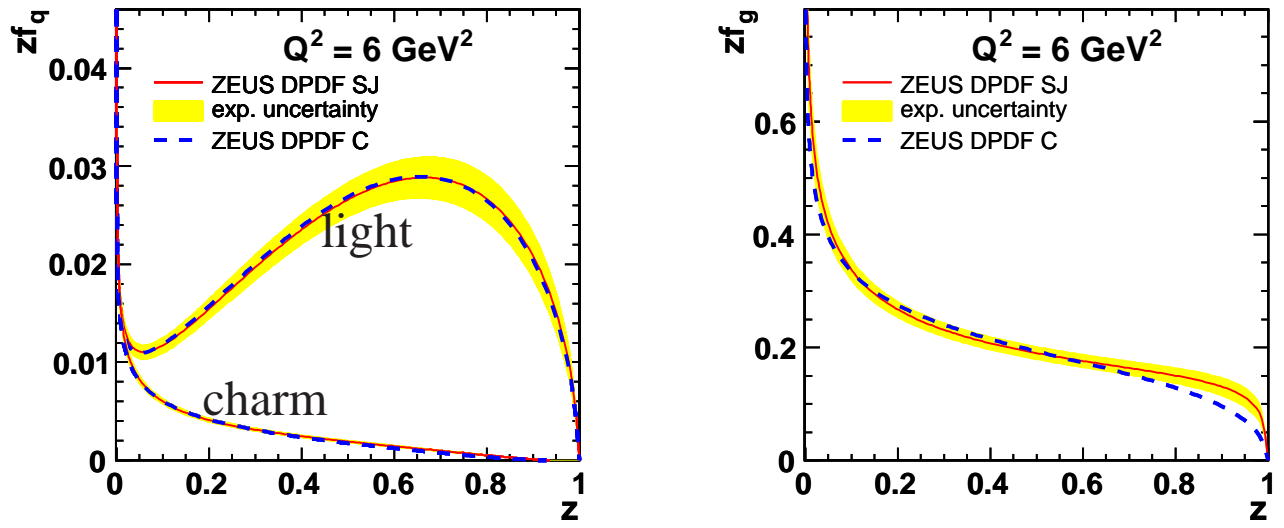

Figure 6: Diffractive quark (left) and gluon (right) parton distributions extracted from a ZEUS fit to inclusive DDIS and diffractive dijet data [20].

They are closely related to the multiple parton interactions discussed in section 5 and are usually quantified by a 'rapidity gap survival probability'. The gap survival probability is expected to be smaller at the LHC than at the Tevatron. First LHC results are now emerging [24], as shown in figure 8. Despite large uncertainties in the predictions, the data cannot be described merely by hadronisation fluctuations in non-diffractive processes and models based on HERA DPDFs without suppression factors clearly overestimate the cross section. The data have been interpreted in terms of a gap survival probability of $0.08 \pm 0.04$, which is a little larger than expected. Measurements using proton-tagged data at the LHC are eagerly awaited in order to suppress the poorly understood 

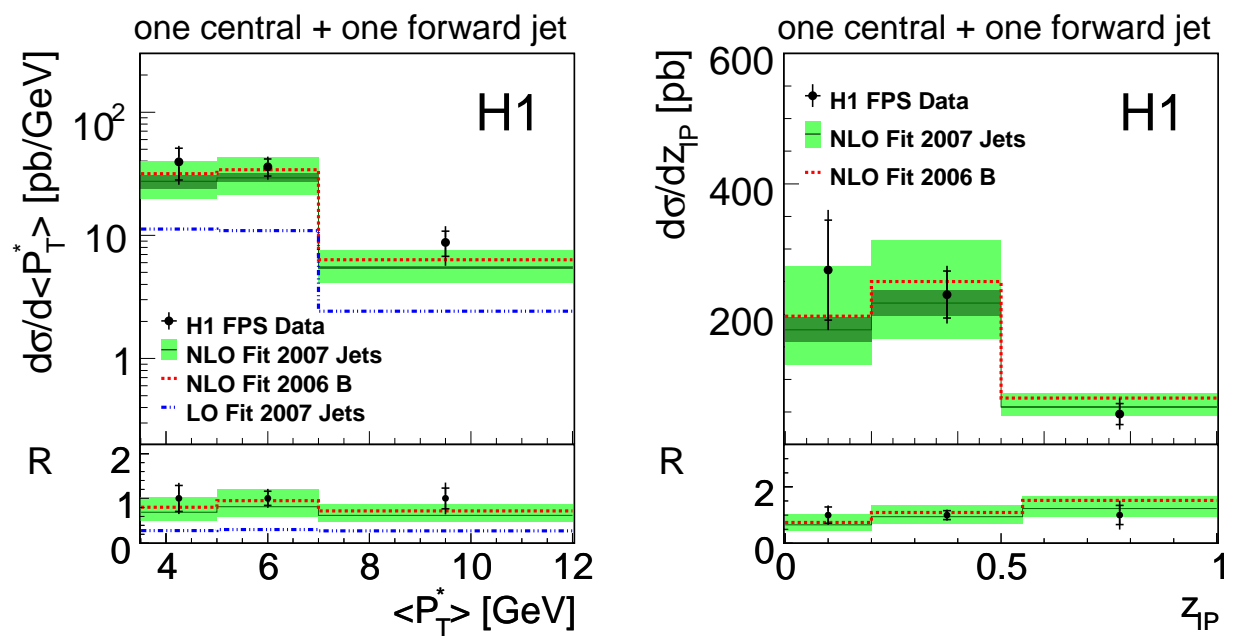

Figure 7: Cross sections for the diffractive production of two jets, at least one of which must be forward of $\eta=1$ in DIS at HERA [22], obtained from events in which an intact leading proton is detected directly. (a) Dependence on the mean jet transverse momentum. (b) Dependence on a hadron level estimator of the momentum fraction of the incoming parton from the DPDFs.

non-diffractive contributions to such distributions.

\section{Non-Diffractive Processes}

Non-diffractive processes under minimum bias conditions are notoriously difficult to understand and to model. Couplings are large, such that perturbative tools are not available and many subtle and inter-connected effects are at play. There were many presentations at ICHEP' 12 which reported measurements of identified and unidentified particle distributions and energy flows, all of which are well suited to the development and tuning of Monte Carlo models which aim to give as complete a description as possible of minimum bias processes. Here a handful of illustrative examples are chosen, particularly in areas where substantial progress has recently been made.

The complexity of the situation and the long range nature of the colour-connections in the final state is illustrated by a recent ATLAS measurement showing a correlation coefficient of larger than $50 \%$ between the low transverse momentum charged particle multiplicities at $\eta=+2.5$ and $\eta=-2.5$ [26]. Another illustration is shown in figure 9, in the form of measurements by several collaborations of the ratio of antiproton to proton yields as a function of rapidity distance $\Delta y$ from the outgoing proton beam particles. At very large $\Delta y \sim 10$, corresponding to the most central region of the detector in the LHC case, no influence from the beam particle is expected and the proton and antiproton rates are compatible, yielding a ratio close to unity. However, the ratio falls significantly below 1 already for $\Delta y \simeq 5$, indicating that the baryon number associated with the beam proton can be transported over remarkably large distances.

An area where progress has been impressively rapid since the turn-on of the LHC is the understanding of the 'underlying event' corresponding to all of the activity in hadronic scattering processes beyond that of the hardest scattering. The sources of the underlying event are the remnants of the colliding hadrons and any activity from multiple scatterings in the same event. The 


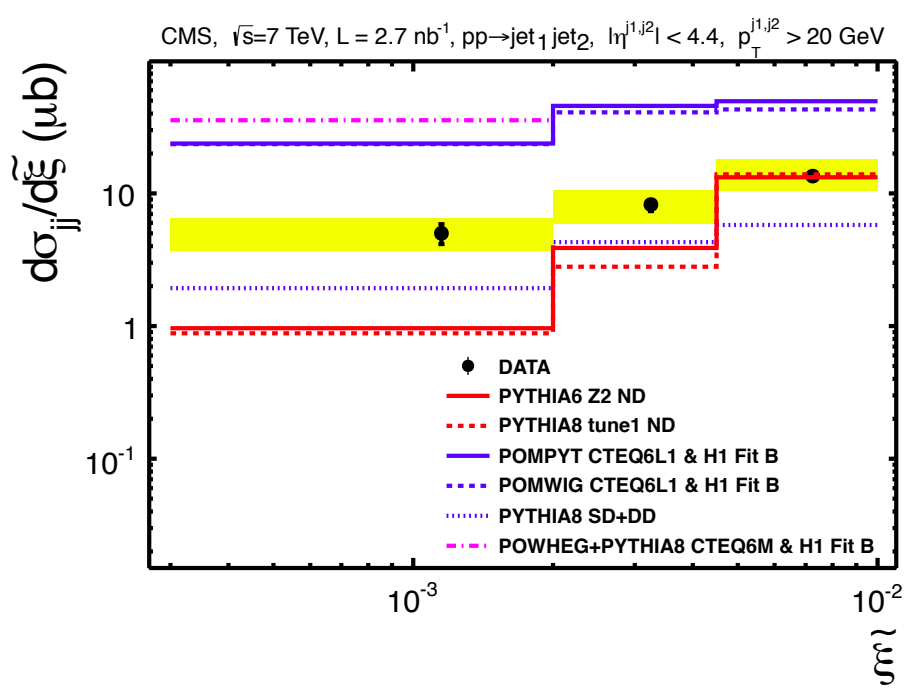

Figure 8: CMS Measurement of the cross section for the production of pairs of jets in LHC events containing rapidity gaps (the variable $\tilde{\xi}$ corresponds to $\xi$ under the interpretation of a diffractive production mechanism). Among others, the data are compared with predictions based on DPDFs from HERA (POMPYT, POMWIG) and with models in which the gaps are produced solely through large fluctuations in the rapidity distribution of final state particles in non-diffractive events (PYTHIA6 Z2, PYTHIA8 tune 1). Figure from [24].

latter are usually modelled on the basis of an integral over different impact parameters between the two hadrons, for each of which secondary partonic scattering is generated on the basis of the proton PDFs at a suitably reduced momentum fraction distributed over an appropriate spatial matter distribution. Constraints on the underlying event have been obtained mainly by studying the properties of charged particles transverse in azimuth to the direction of the hard scatter, as estimated by the direction of the highest transverse momentum track. The arrival of the first data on such observables from the LHC quickly showed that the existing models were inadequate, giving rise to an intense tuning effort. The state of the art in this regard is represented by the ' $\mathrm{Z} 1$ ' tune of PYTHIA6 and other closely related tunes [27]. This has been benchmarked using a variety of observables, an example of which is shown in figure 10 . Here, the $\eta$ and $\phi$-averaged density of charged particles in the transverse region is shown as a function of the transverse momentum of the leading track. In addition to LHC data at $\sqrt{ } s=7 \mathrm{TeV}$, data from the Tevatron at the nominal $\sqrt{ } s=1.96 \mathrm{TeV}$ and from a Tevatron energy scan at $\sqrt{ } s=900 \mathrm{GeV}$ and $\sqrt{ } s=300 \mathrm{GeV}$ are also shown. The data are well described by the Z1 tune throughout. The good description of the energy dependence bodes well for a high quality early description of LHC data when the energy increases to $\sqrt{ } s=13$ or $14 \mathrm{TeV}$ from 2015 . There are many other examples of successful underlying event predictions by similarly tuned Monte Carlo models, using a variety of dedicated samples involving for example dijet or Drell-Yan dimuon production, which provide natural hard scales and a clearer direction for the hard interaction [28]. The models also give a good description of alternative global event characterisation variables, including event shapes such as thrust [26].

Underlying event studies are usually only sensitive to particle production in the central region, 


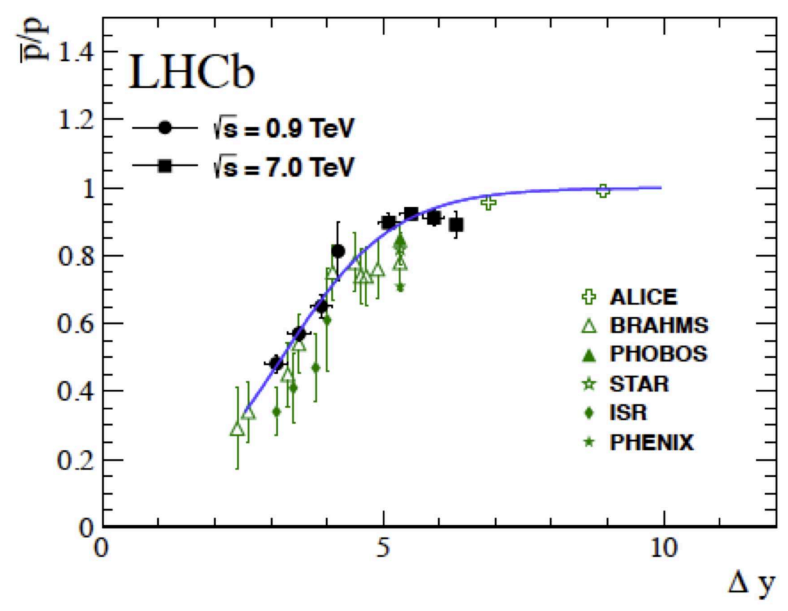

Figure 9: Compilation of data on the ratio of production rates of antiprotons to protons as a function of the rapidity distance $\Delta y$ from the outgoing proton beam particles [25].

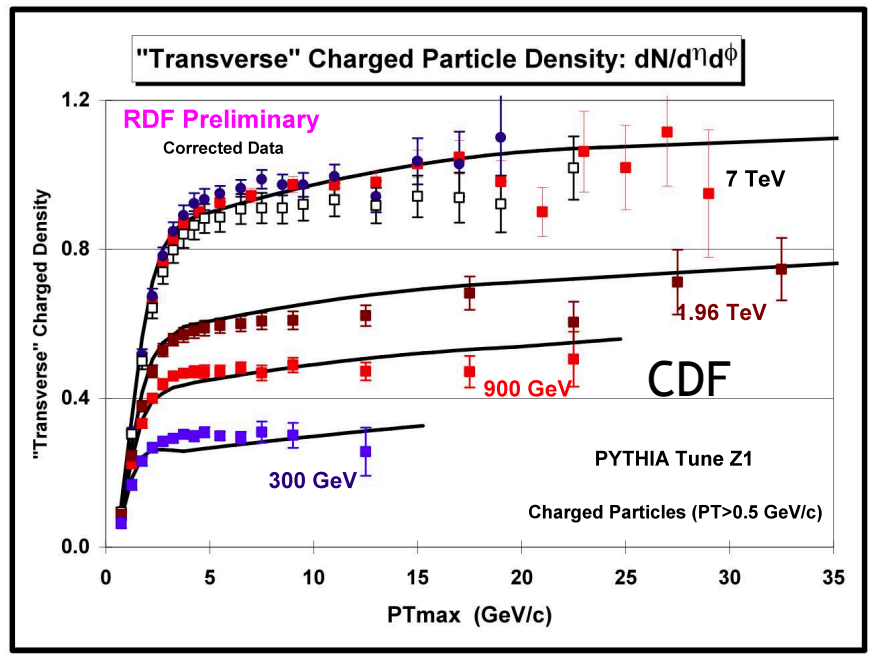

Figure 10: Charged particle density, $\mathrm{d} N /(\mathrm{d} \eta \mathrm{d} \phi)$ in a region transverse to the leading track in minimum bias samples, as a function of the leading track transverse momentum, $P_{\mathrm{T} \text {, max }}$. The $\sqrt{ } s=7 \mathrm{TeV}$ data were recorded by ATLAS (blue circles), CMS (red squares) and ALICE (open squares). Figure from [27].

where the best charged particle tracking is located. Another area where there has been considerable recent experimental progress is in measurements of particle production and energy flow in the forward direction. As illustrated in figure 11, the LHC experiments between them are sensitive over a very wide range, extending well beyond the rapidity plateau, with charged particle production in addition to calorimeter coverage.

An intensive effort has been made to obtain data extending as forward in rapidity as possible $[26,28,29,31]$. An illustrative example is shown in figure 12, where the final state transverse energy density is measured to $|\eta|=4.9$. Whereas the standard tunes of PYTHIA are successful in the central region, they lie further and further below the data as $|\eta|$ increases. This discrepancy may 


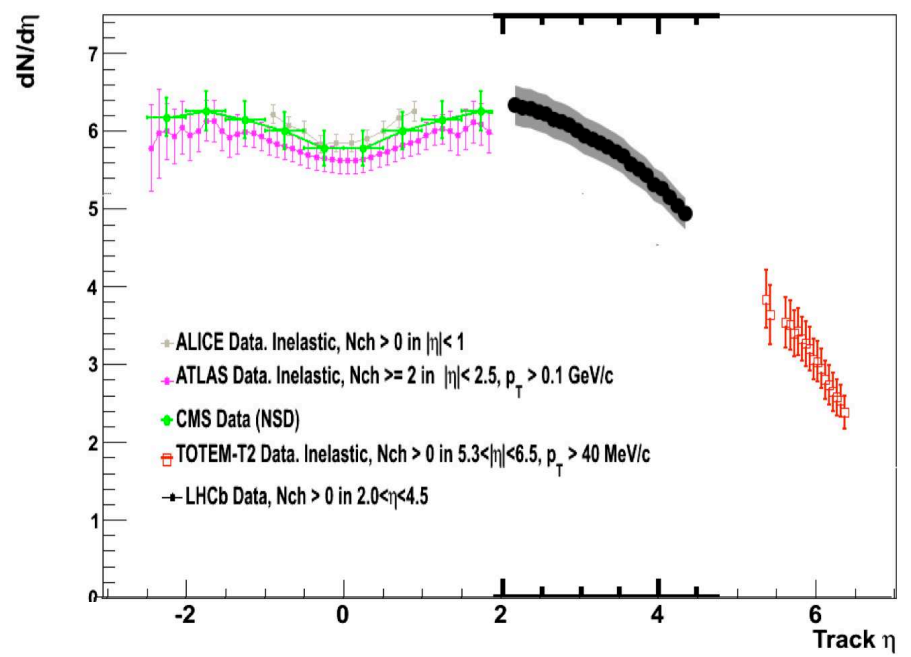

Figure 11: Compilation of LHC charged track multiplicity data as a function of pseudorapidity [29].

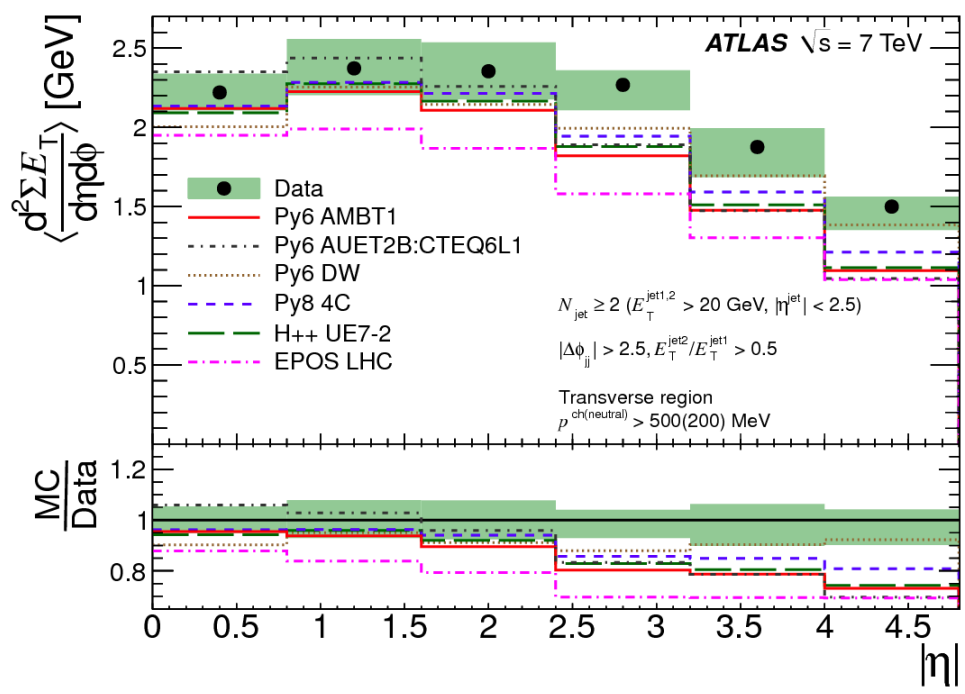

Figure 12: ATLAS transverse energy density for minimum bias $\sqrt{ } s=7 \mathrm{TeV}$ LHC $p p$ collisions, averaged over azimuthal angle and the indicated intervals of peseudorapidity [30].

be explained by a combination of inadequate modelling of the low $x$ gluon density of the proton, the treatment of diffractive channels, the underlying event and parton cascade dynamics. Predictions based on dedicated models of cosmic air showers such as EPOS [32] are more successful. Similar conclusions are reached in the other related studies which extend even further forward and also address the $\sqrt{ } s$ dependence $[33,34,35,36]$.

\section{Summary}

The introduction of new data in a previously unexplored energy regime at the LHC has given 
new motivations and a renewed stimulus for the reinvestigation of the softest processes taking place in hadronic scattering. In addition to the impressive precision obtained, the introduction of new observables and the investigation of extended kinematic regions has given complementary insights. Together with continuing high quality measurements from HERA and the Tevatron, this has led to a deeper understanding of long-standing problems such as the underlying dynamics of multi-parton interactions and of diffractive processes, as well as increasingly reliable tools for a complete model of hadronic physics at the $\mathrm{TeV}$ scale.

\section{Acknowledgements}

It was a great privilege to attend the conference and to give a plenary talk - sincere thanks to the organisers for the invitation. For help in preparing the talk or for supplying material, I would also like to thank G. Alves, S. Bhadra, R. Cieselki, M. Diele, R. Field, C. Glasman, A. Grebenyuk, M. Klein, T. Martin, R. Muresan, H. Niewiadoniski, R. Polifka, D. Salek, A. Soffer and V. Simak.

\section{References}

[1] Banjo Paterson 'Waltzing Matilda' (1895).

[2] T. Sjostrand, S. Mrenna and P. Skands, JHEP 0605 (2006) 026 [hep-ph/0603175].

[3] T. Sjostrand, S. Mrenna and P. Skands, Comput. Phys. Commun. 178 (2008) 852 [arXiv:0710.3820 [hep-ph]].

[4] R. Engel, Z. Phys. C 66 (1995) 203.

[5] O. Aharony, Phys. Rept. 323 (2000) 183 [hep-th/9905111].

[6] Z. Ajaltouni et al., 'Proceedings of HERA and the LHC workshop series', chapter 5 [arXiv:0903.3861 [hep-ph]].

[7] Slides available at epweb2.ph.bham.ac.uk/user/newman/diffraction/ICHEP12-Plenary.pdf

[8] D0 Collaboration, Phys. Rev. D 86 (2012) 012009 [arXiv:1206.0687 [hep-ex]].

[9] TOTEM Collaboration, 'Measurement of proton-proton elastic scattering and total cross-section at $\sqrt{ } s=7 \mathrm{TeV}$ ', CERN-PH-EP-2012-239.

[10] TOTEM Collaboration, Europhys. Lett. 95 (2011) 41001 [arXiv:1110.1385 [hep-ex]].

[11] TOTEM Collaboration, Europhys. Lett. 96 (2011) 21002 [arXiv:1110.1395 [hep-ex]].

[12] TOTEM Collaboration, 'A luminosity-independent measurement of the proton-proton total cross-section at $\sqrt{ } s=8 \mathrm{TeV}$ ', CERN-PH-EP-2012-354.

[13] ZEUS Collaboration, Phys. Lett. B 708 (2012) 14 [arXiv:1111.2133 [hep-ex]].

[14] ATLAS Collaboration, Nature Commun. 2 (2011) 463 [arXiv:1104.0326 [hep-ex]].

[15] ALICE Collaboration, [arXiv:1208.4968 [hep-ex]].

[16] CMS Collaboration, [arXiv:1210.6718 [hep-ex]].

[17] ATLAS Collaboration, Eur. Phys. J. C 72 (2012) 1926 [arXiv:1201.2808 [hep-ex]]. 
[18] M. Ryskin, A. Martin and V. Khoze, Eur. Phys. J. C 72 (2012) 1937 [arXiv:1201.6298 [hep-ph]].

[19] H1 and ZEUS Collaborations, Eur. Phys. J. C 72 (2012) 2175 [arXiv:1207.4864 [hep-ex]].

[20] ZEUS Collaboration, Nucl. Phys. B 831 (2010) 1 [arXiv:0911.4119 [hep-ex]].

[21] H1 Collaboration, Eur. Phys. J. C 48 (2006) 715 [hep-ex/0606004].

[22] H1 Collaboration, Eur. Phys. J. C 72 (2012) 1970 [arXiv:1111.0584 [hep-ex]].

[23] CDF Collaboration, Phys. Rev. Lett. 84 (2000) 5043.

[24] CMS Collaboration, Phys. Rev. D 87 (2013) 012006 [arXiv:1209.1805 [hep-ex]].

[25] LHCb Collaboration, 'Measurement of the $\bar{p} / p$ ratio in $L H C b$ at $\sqrt{ } s=900 \mathrm{GeV}$ and $7 \mathrm{TeV}$ ', LHCb-CONF-2010-009.

[26] T. Martin, 'ATLAS studies of diffraction, soft particle production and double parton scattering', parallel session talk at this conference.

[27] R. Field, 'The Energy Dependence of the Underlying Event in Hadron-Hadron Collisions', parallel session talk at this conference.

[28] G. Alves, 'Forward Physics Results from CMS', parallel session talk at this conference.

[29] H. Niewiadomski 'New measurements of Forward Physics in the TOTEM Experiment at the LHC', parallel session talk at this conference.

[30] ATLAS Collaboration, JHEP 1211 (2012) 033 [arXiv:1208.6256 [hep-ex]].

[31] R. Muresan 'Studies of soft $Q C D$ at $\mathrm{LHCb}$ ', parallel session talk at this conference.

[32] K. Werner, Nucl. Phys. Proc. Suppl. 175-176 (2008) 81.

[33] CMS Collaboration, arXiv:1302.2394 [hep-ex].

[34] TOTEM Collaboration, Europhys. Lett. 98 (2012) 31002 [arXiv:1205.4105 [hep-ex]].

[35] CMS Collaboration, JHEP 1111 (2011) 148 [Erratum-ibid. 1202 (2012) 055] [arXiv:1110.0211 [hep-ex]].

[36] LHCb Collaboration, Eur. Phys. J. C 72 (2012) 1947 [arXiv:1112.4592 [hep-ex]]. 